\title{
Exploring Mount Erebus by Walking Robot
}

\section{David Wettergreen, Chuck Thorpe, and Red Whittaker}

The Robotics Institute, Carnegie Mellon University

Dante is a tethered walking robot capable of climbing steep slopes. In 1992 it was created at Carnegie Mellon University and deployed in Antarctica to explore an active volcano, Mount Erebus. The Dante project's robot science objectives were to demonstrate a real exploration mission, rough terrain locomotion, environmental survival, and selfsustained operation in the harsh Antarctic climate. The volcano science objective was to study the unique convecting magma lake inside Mount Erebus' inner crater. The expedition demonstrated the advancing state-of-art in mobile robotics and the future potential of robotic explorers. This paper details our objectives, describes the Dante robot, overviews what happened on the expedition and discusses what did and didn't work.

We would like to acknowledge the people whose contributions made Dante and the expedition to Mount Erebus possible. The mechanism was designed and assembled by Eric Hoffman of $\mathrm{K}^{2} \mathrm{~T}$ Inc., Matt Arnold, Tad Dockstader, and Dimitrios Apostolopoulous. The electronics were fabricated by Bryon Smith, Dan Christian and Scott Boehmke. Paul Keller, Jay West, Chris Fedor, Bill Ross, Dan Christian, and Henning Pangels implemented software so that Dante could sense, plan, communicate, and walk. Leslie Thorpe sewed weatherproof covers. Chuck Whittaker of RedZone Robotics Inc., Rob McWilliams, and Jim Osborn managed the project. Jim Martin, Gary Baun, Brian Albrecht, Jim Frazier, Bob Smith and others at Carnegie Mellon provided critical assistance whenever it was needed. The Erebus expedition group included Red Whittaker, David Wettergreen and Dan Christian from Carnegie Mellon, Eric Hoffman from $\mathrm{K}^{2} \mathrm{~T}$ Inc., Phil Kyle, Bill McIntosh, Nelia Dunbar and Ken Sims from New Mexico Institute of Mining and Technology, and David Lavery, and Steve Thompson from NASA.

\section{Introduction}

In 1992 a walking robot named Dante ${ }^{1}$ was designed and built at Carnegie Mellon University. Using a tensioned tether, Dante can ascend and descend steep slopes. It is designed to rappel into and explore active volcanic craters. The Dante project was an ambitious attempt to proceed, in 10 months, from idea to implementation. The culmination was an expedition to an active volcano, Mount Erebus, in Antarctica.

In terms of robot science, the objectives were to demonstrate a real exploration mission, rough terrain locomotion, environmental survival, and selfsustained operation in the cold, windy, bright, rugged Antarctic environment. The expedition to Mount Erebus was indeed a real mission. Dante climbed into the steep sided crater, surviving the environment and operating in a self-sustaining manner until the failure of a critical component, the communications tether, necessitated rescue and the premature termination of the crater exploration.

The volcano science objectives were also real and involved the study of Mount Erebus' unique convecting magma lake. Dante was equipped with instruments to determine the chemical and isotopic composition of gas generated by the volcano's magma lake, to measure the radioactivity of materials near the lake and to measure the temperature of the magma itself.

Dante can rappel up and down steep slopes and surmount obstacles as large as 1 meter in height. It can perceive and model the terrain around it using a scanning laser rangefinder and a trinocular camera system. Its planning software determines safe paths and adjusts the gait to avoid obstacles. While some of its computers reside off-board at a base station and are linked via a fiber optic cable, Dante is computationally self-sufficient, able to chart its own course, react to perceived terrain, and acquire data from science payload sensors. Dante's eight pantographic legs are organized in frames of four-an inner frame and an outer frame-with each coupled through a drivetrain that provides an intrinsic walking motion. To walk, four legs simultaneously lift

1. The robot is named Dante in reference to the poem, The Divine Comedy by Dante Alegheri, in which Dante travels to the underworld. Erebus is a cloud of mist that obscures the entry to hell. Early mission scenarios also had a transport robot named Virgil for the Roman poet who guides Dante during his quest. In keeping with the theme, the cart used to carry Dante around is called Geryon after a flying daemon who gave Dante a lift. 
and reach forward while the other four supporting legs propel the body. Each of the legs can individually adjust its height to avoid obstacles in the terrain. On steep slopes the tensioned tether provides a reactive force to gravity, assists in maintaining equilibrium, and allows Dante to rappel like a mountain climber

This paper briefly introduces our objectives and Mount Erebus, describes the Dante robot, overviews the software system that enabled Dante to walk, and then describes what happened on Erebus and what we learned.

\section{Robot Science}

\section{Objectives}

There are a number of technical challenges in designing a robot that can rappel into the extreme terrain and environment of a volcanic crater and survive. These challenges aligned with our objective to show that a robot explorer could perform an actual exploration mission, that it could handle the terrain and the environment and that it could sustain itself. We wished to demonstrate that a walking robot could traverse the rough terrain and steep slopes typified by volcanic craters, something beyond what had been or could be shown in the controlled setting of a laboratory. The terrain of a volcanic crater requires that all we have learned about legged locomotion be put into practice. In addition, the steepness of the slope requires the addition of a tensioned tether for support. The coordination of this element adds complexity but further enhances capability of the explorer.

Antarctica presents environmental challenges unmet by robotic explorers. The low temperature, high wind force, intense brightness, and extreme terrain are daunting. This severe setting forces issues of environmental survival as well as self-sustained operation. To be viable, an explorer must be to be rugged and reliable. We wished to take on the difficulties of ruggedizing the robot to environmental in order to further the reliability of robots in the field. The survival and success of an extended robotic exploration in Antarctica lends credibility to the use of robotic explorers and advances the stateof-art for terrestrial, lunar and planetary missions.

Although it is a prototype, Dante is not a laboratory device-it is a field-worthy robot. Its components are selected to withstand the harsh environment inside a volcano. Its enclosures are sealed and heated. Its lenses are coated against the acidic plume. The metallic surfaces are anodized to harden them. The computing is specially rated for extreme cold. In terms of survival, Dante's weakness may be that building it fast and cheap came at the expense of redundancy. The design approach was to choose single solutions, do the best design possible, make it reliable, and have very few redundant components. Such a strategy is also dictated when system weight is a guiding concern as the logistics of an expedition made it this time. It is a weakness because it means that single points of system failure will exist.

An essential feature of an explorer is its ability to sustain itself for extended periods of time. Our goal was to embody Dante with sufficient perceptive and planing ability to sustain operation. Dante is equipped with perceptive sensors so that it can sense its and environment and proprioceptive sensors so that it can sense itself. It has on-board computing to provide it with basic capabilities for motion. It has sufficient sensing in its feet alone to allow it to grope through the terrain blindly. Using its perception and planning abilities Dante can walk autonomously. Without much direction from operators, it should be able to keep going in all but the most difficult of situations.

\section{Scenario}

An untethered robotic device that can climb sheer cliff walls is well beyond the state-of-art. We determined the safest and most robust method for exploring a crater would be to rappel down the rock face as a human climber would. Early in the project, we envisioned the following scenario.

At the beginning of the deployment, Dante would be transported to the crater rim. In order to descend the steep walls, Dante's tether, anchored at the rim, would remain under tension to support much or all of the robot's weight just like a climbing rope. The terrain outside the crater, between the mountainside control station and the summit, might consist of patches of snow, large boulders and much rock debris comparable to a plowed field. A fiber optic line would be strung between the summit and the station across this terrain. In order to reduce weight and to allow for long-duration operation, power and high level computing would remain off the robot and be transmitted to the robot via the tether.

The robot would descend the crater wall unreeling tether as it proceeded. The crater walls may have slopes between $50^{\circ}-90^{\circ}$ and be composed of both ice and rock. These walls would have embedded lava bombs and possibly fumarole fed ice towers that must be negotiated. We hoped to find a descent route of approximately $75^{\circ}$ that would drop from 
the rim all the way to the bottom of the crater without any major obstructions or overhangs. For much of the mission Dante would be teleoperated by researchers giving it short commands to walk, or to roll, pitch, yaw, or lift (or lower) while standing in place. The researchers would use on-board cameras including an overview pan-tilt camera to view the surrounding terrain. Dante would carry terrain sensors including a trinocular stereo system and a laser range-finder which it can use to automatically perceive and model the terrain. For some portion of the mission we hoped to turn Dante loose, to autonomously negotiate the crater wall by sensing the local terrain and planning its own actions.

As it descended into the crater Dante would perform remote sensing operations, collect samples, and make photographic records. Although the stated objective was to reach the bottom, much of the scientific agenda would be accomplished during the ascent/decent.

The robot may have to cross substantial transitions in slope during its descent. These transitions might involve coming off the wall of the crater and crossing level terrain to the edge of another drop. At these edges walls may drop steeply, $80^{\circ}-90^{\circ}$, down into the crater. Near the bottom of the inner crater the robot must transition to rough but level terrain where volcanologists would direct it to areas of interest. On the crater floor the robot would study of the unique convecting magma lake and acquire samples of gases and particulates.

This descent process would be reversed to return to the summit - the ascent path is dictated by the position of the tether during the descent. The robot would transition from the crater floor to the steep walls of the crater. It must climb up these, transition to the changes in slope, and finally pick its way back to the rim.

\section{Volcano Science}

\section{Mount Erebus}

Mount Erebus, situated at $77.53^{\circ}$ south latitude and $167.15^{\circ}$ east longitude, is the only active surface volcano in Antarctica. In Figure 1, an ancient side vent is in the front, the main crater is behind, and the robot control station which was established for Dante is $2 \mathrm{~km}$ away in the direction of Fang ridge which is in the left rear. Erebus rises from the Ross Ice Shelf on the East Antarctic coast, forming the main peak of Ross Island.

The summit of Mount Erebus is $3794 \mathrm{~m}(12447 \mathrm{ft})$ in height, although the apparent altitude is higher of because atmospheric pressure decreases near the

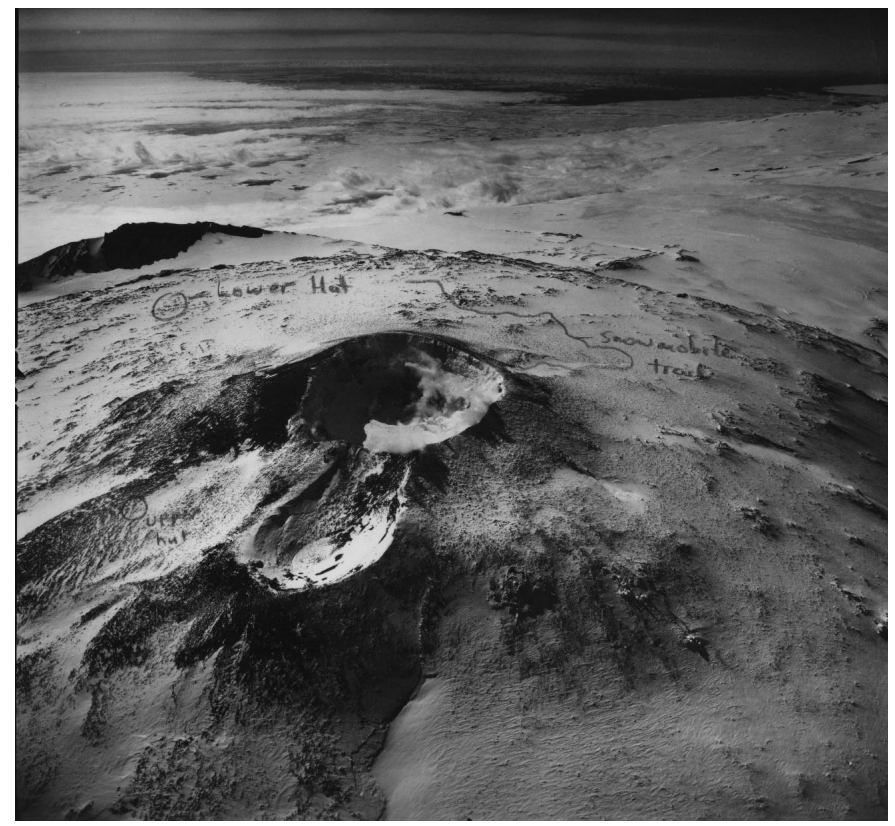

Figure 1: Aerial view of Mount Erebus

Earth's poles. The summit of Erebus opens into a main crater, a $500 \mathrm{~m}$ by $600 \mathrm{~m}$ ovoid, approximately $150 \mathrm{~m}$ deep. This outer main crater contains an inner crater (approximately $200 \mathrm{~m}$ in diameter and an additional $100 \mathrm{~m}$ deep) and an area of active gassing fumaroles. In Figure 1, the inner crater is visible within the outer main crater. The inner crater contains more fumaroles and a convecting magma lake. A magma lake is a rare example of volcanic equilibrium. Fresh magma from deep inside the earth rises to the surface of the lake, releases gases and, having cooled, sinks down without violently erupting.

The crater walls (interior) have a $75^{\circ}-90^{\circ}$ slope and

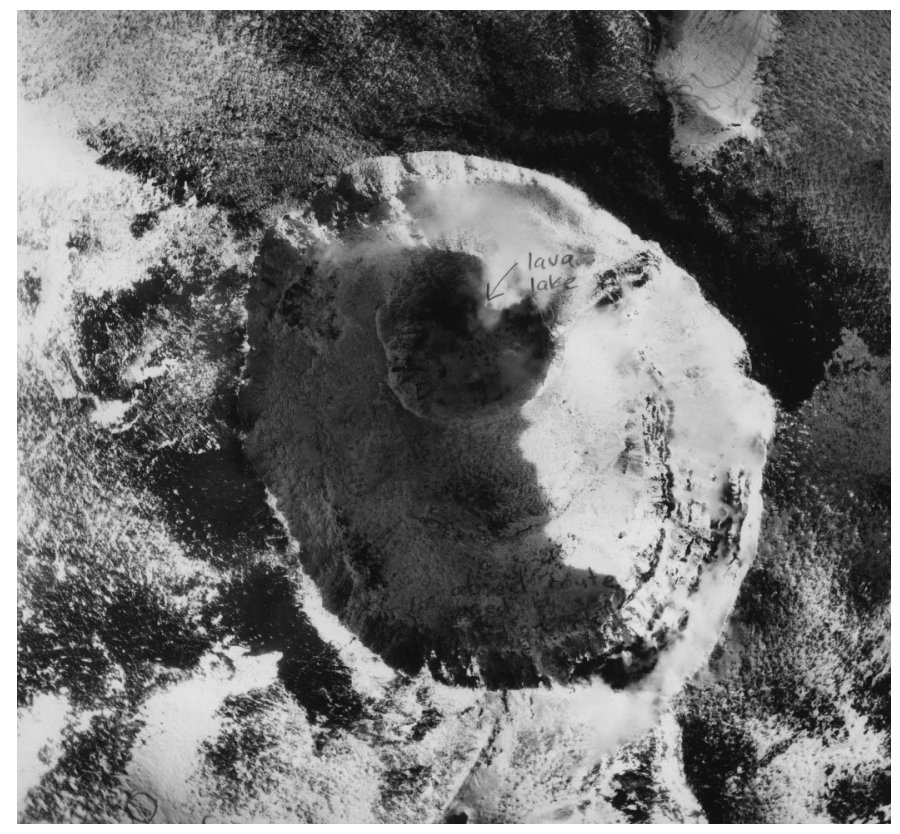

Figure 2: Top view of Erebus' crater showing the inner crater 
are primarily exposed, solidified, igneous rock with some areas of ice and snow. Some areas near the rim are composed of looser material at a $50^{\circ}$ slope. Fumaroles in the crater walls create shelves and formations of ice that grow outward from the walls. To reach the crater floor it is necessary to navigate over or around these obstacles.

Even during the Austral summer, clear skies seldom last more than a day. There is good visibility on the rim about $50 \%$ of the time, but the inner crater floor is obscured at least $90 \%$ of the time because winds across the summit hold the plume down in the crater making visibility from the rim to the floor a rare event.

Although it is the most arid continent on earthsome areas may not have had precipitation for thousands of years - the humidity inside the crater can be very high, so the possibility of condensation on equipment exists. Perhaps more critical to a well sealed system, is the presence of acids, $\mathrm{HF}, \mathrm{HCl}$, and $\mathrm{H}_{2} \mathrm{~S}$, in the plume.

The typical summer wind on Erebus is 10-20 kilometers per hour. It does not snow in great quantities but the wind can create white-outs by suspending highly-specular ice particles in the air. Such a white-out can intensify brightness, reduce visibility, make shadows disappear and cause features to become indistinguishable.

The summer temperature on Erebus ranges from $-50^{\circ} \mathrm{C}$ to $-10^{\circ} \mathrm{C}$. The median seems to be around $-20^{\circ} \mathrm{C}$. The temperature increases when close to thermal sources - the magma lake and high-pressure fumaroles are believed to be hotter than $600^{\circ} \mathrm{C}$-but the temperature gradient is such that approach to within a few meters is possible.

\section{Objectives}

The scientific importance of exploring Mount Erebus lies in its uniqueness. Erebus, in addition to being a rare Antarctic volcano, contains one of only three known convecting magma lakes, in which fresh magma from deep inside the earth rises, degases, cools and sinks in a continuous convection cycle. The lake releases Chlorine, Fluorine and Sulfur into the Antarctic atmosphere in significant quantities.[1] The composition and concentration of the gases and the information they provide about the composition of magma is of great interest to geophysical, geochemical and atmospheric scientists. Information about gases and particles released by any active volcano is extremely rare because of the dangers to humans involved in collecting samples.
The principle objective in entering the volcano is to obtain pristine gas samples from the area of the convecting magma lake. It is essential that the gas be sampled from the magma before it cools and undergoes chemical changes. The convection currents inside Erebus insure fresh magma arrives at the surface. Such pristine gas samples, taken at high temperature so that no materials sublimate, are important because they reveal much about the processes deep inside the magma chamber. For example, a sample may contain isotopes of hydrogen and oxygen that are in a ratio of $\mathrm{H}^{2}$ (deuterium) to $\mathrm{O}^{16}$ and $\mathrm{O}^{18}$ that is different from what is predicted by geochemical models. Insights into the composition and nature of magma within the Earth's mantle are possible by studying this magma lake.

Gas and filter samples have been taken from Erebus' rim but no gas or aerosol samples have been acquired close to the source. By identifying the composition of gases released from the magma lake and nearby vents, the effect of venting and erupting volcanos on the atmosphere can be better understood. Atmospheric scientists are specifically interested in the types and quantities of gases released into the Antarctic environment. The plume disperses across the East Antarctic ice sheet spreading $\mathrm{Cl}$ and $\mathrm{F}$. The amount of these elements released may be directly correlated to the amount deposited in the ice revealing a historic record of volcanic activity.

\section{Dante Hardware}

In comparison to wheeled mechanisms, legged mechanisms require complex design, move slowly, and are difficult to control. However, for locomotion over rough or discontinuous terrain such as the crater of a volcano, legged mechanisms are potentially superior to wheeled mechanisms. Legged mechanisms make discrete terrain contacts and avoid undesirable footholds and large discontinuities while wheeled mechanisms have rollers in continuous contact with the ground. The posture of most wheeled mechanisms is dependent upon the terrain, but a legged mechanism can isolate its body from the terrain.[2] By adjusting its legs and the tension on the tether, a rappelling walker can achieve a stable stance and smooth motion even on nearly vertical inclines. We believe that a legged robot is well suited to the task of carefully rappelling down into a volcanic crater.

\section{Mechanism, Electronics, and Sensors}

Dante is an eight-legged robot designed for rappelling down the crater wall and carrying a payload of 
scientific instruments into the volcano. In Figure 3, Dante is shown on level ground. The legs of the outer frame are raised. The eight lightly shaded boxes are the actuators for the vertical motion of the legs. It is approximately $3 \mathrm{~m}$ in length, $2 \mathrm{~m}$ in width,

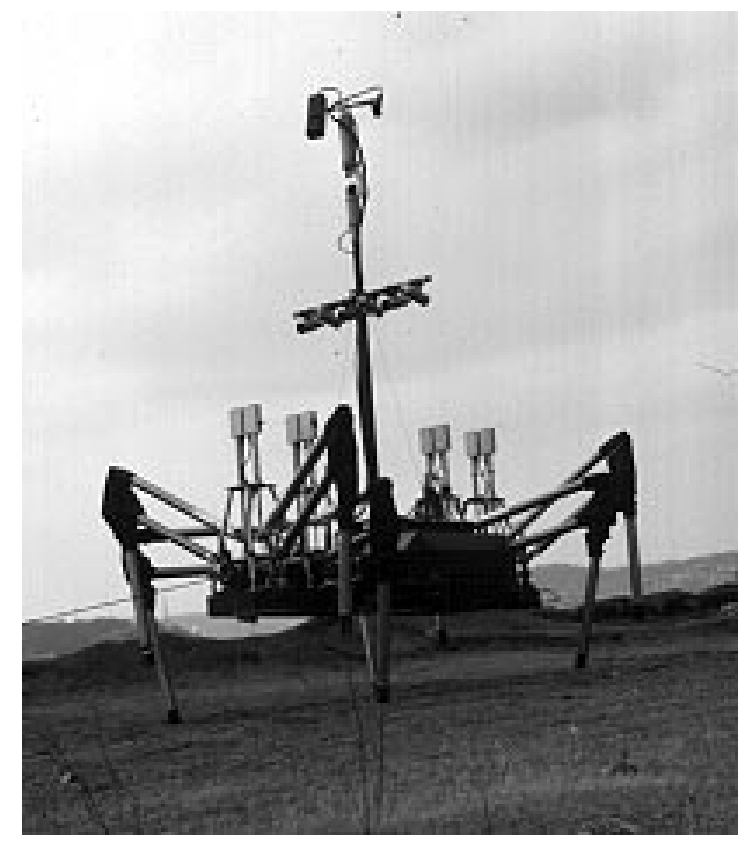

Figure 3: Dante

and $400 \mathrm{~kg}$ in mass. It is composed of two frames, eight legs, two enclosures (one for electronics and one for the science payload) a tether reeling mechanism and a sensor mast.

The eight pantographic legs are arranged in two groups of four on an inner and an outer frame. A single drive motor and drivetrain on each frame drives all four legs on that frame. Unique four-bar linkages on each leg mechanically convert a rotational motion into a stepping motion. (See Figure 4.) The motion of the four-bar linkage and the motion of the foot, amplified in magnitude by the pantograph is shown in Figure 5. This mechanical coupling resulting from driving all four legs of a frame together reduces external force loops and

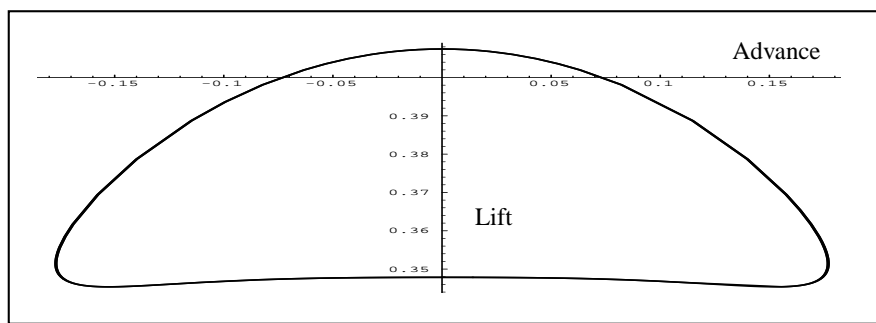

Figure 5: Motion of the foot mechanically generated by the fourbar linkage

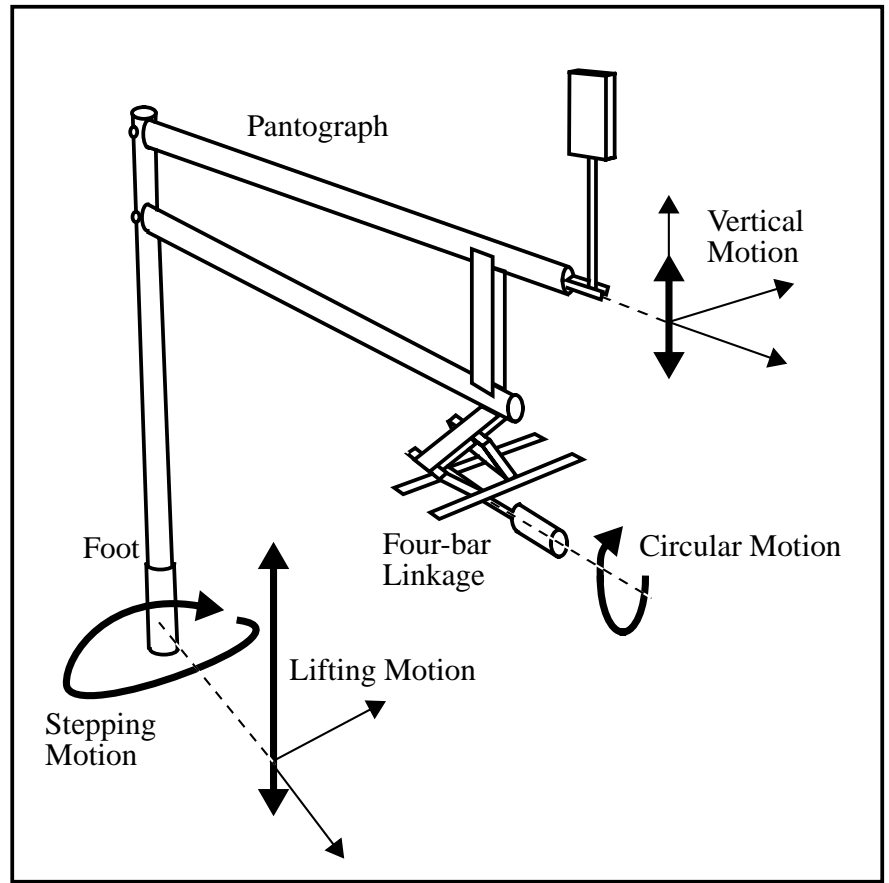

Figure 4: Dante's pantographic leg coupled to a vertical actuator to lift the feet and to a four-bar linkage for an intrinsic stepping motion.

enables an intrinsic gait with only one motion. To walk, four legs simultaneously lift and reach forward while the four supporting legs propel the body. To attain an overlap in the support phase during which all legs are on the ground and to allow smooth transition at the beginning and end of each frame recovery, the motion control software drives the four-bar three times faster while the legs are unloaded and recovering forward through the air.

Dante's frames can rotate with respect to each other to change the walker's heading. The maximum rotation per step is very small (about $3^{\circ}$ ) but can be repeated as needed. The approach is to avoid large obstacles well in advance to minimize turing and the lateral forces imposed by the tether.

In addition to the basic stepping motion enabled by the four-bar, each of the legs can individually adjust its height, compounding the stepping motion, in order to avoid obstacles in the terrain and adapt to discontinuities. This is accomplished by a dedicated linear actuators on each leg. Each foot is fitted with capaciflector proximity sensors, which use the distortions in a flexible capacitive film to measure the distance to nearby obstacles. In the sole are a contact switch and a load cell that are used to determine when the foot makes or breaks contact with the ground and to measure the distribution of force among the feet. This group of sensors is sufficient to blindly grope for footfalls with no foreknowledge 


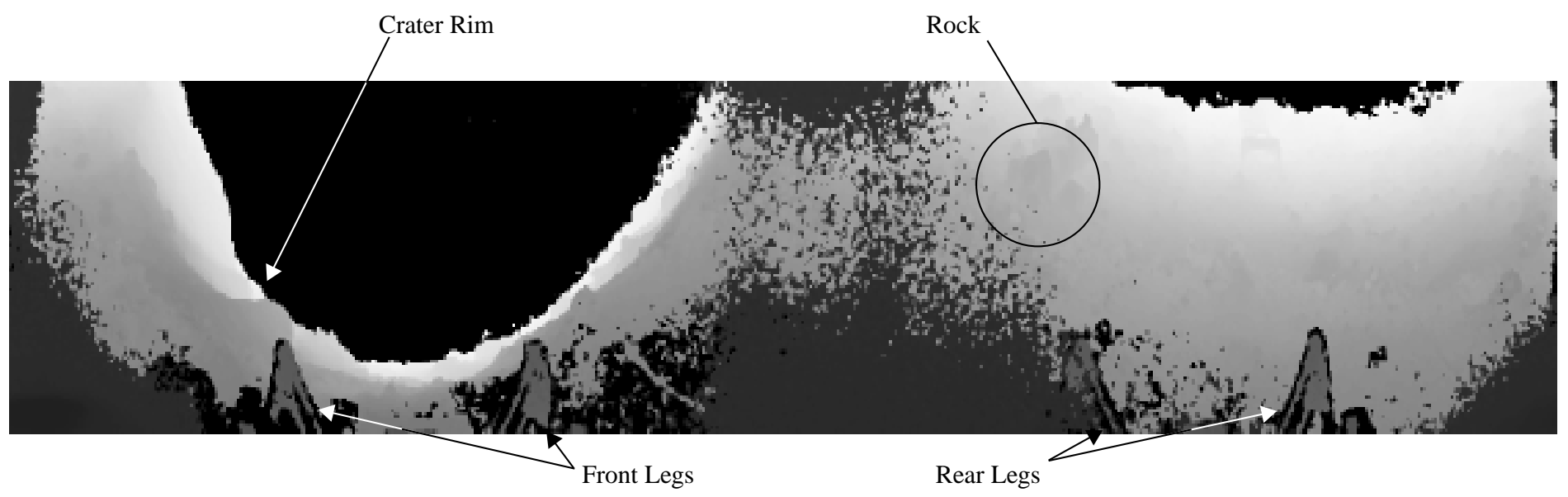

Figure 6: Full $350^{\circ}$ view of laser rangefinder data from Erebus crater rim

of the terrain.

Dante has two perception sensors: a trinocular stereo jig and a scanning laser rangefinder. Refer to Figure 3 to see, at the top of Dante's mast, the teleoperation camera on a pan-tilt platform, beneath which are the laser rangefinder and the transversemounted trinocular stereo jig. The scanning laser rangefinder senses terrain depth in a full circle around Dante. In Figure 6 Dante's front and rear legs are visible and the dark region in the upper left indicates there is nothing in front of Dante-it is standing on the crater rim. The trinocular stereo system has two triplets of cameras for looking forward and looking backward. Each set can capture a triplet of images. Data from these perception sensors can be processed to map the terrain around Dante.

On steep slopes, Dante's tensioned tether provides a reactive force to gravity. The tether reel, mounted in Dante's midsection, has an integral tension measuring system and angle sensors to measure the direction that the tether exits the robot. This knowledge allows characterization of the forces and moments being applied to Dante. The tensioned tether, in addition to providing support with its Kevlar outer weave, delivers power through six thin conductors in its inner weave. Communication to Dante is through a single fiber optic line located at the core of the tether, over which seven video, one ethernet and two serial channels are multiplexed. Radio telemetry, although expensive, is possible but the necessity of a tether provided an available route for communication.

The fiber optic line in Dante's tether is attached to a $2 \mathrm{~km}$ (nonload-bearing) extension to run from the volcano's rim directly to the mountainside control station. In the control station video, ethernet, and serial signals are demultiplexed and sent to control computers and monitors. Two Sun Sparcstations run the perception, planning, simulation and user interface processes. From these workstations Dante's actions are determined and sent to the on-board physical controller. These workstations and compressed video signals can be connected to a NASA TDRSS ground station and sent live to Goddard Space Flight Center (GSFC). Operators at GSFC are able to watch through Dante's cameras and, by connecting to the mountainside computers, can command the robot.

On either side of its midsection Dante has two enclosures. Both are powered, sealed, heated and internally ventilated. One enclosure contains electronics and computing. In it a VME backplane (with dual CPU, motion control, and DAADIO boards), motor amplifiers and telemetry hardware, are housed. The science payload is carried in the other enclosure.

\section{Science}

Dante carries a science payload including a number of physical sampling devices, some of which are paired with on-board real-time devices that can collect continuous data while inside the volcano, something unavailable to date. Pristine gases are obtained by using a long hollow titanium probe that is mounted on the front of the robot and can be positioned with two degrees-of-freedom. The probe is plumbed to a variety of devices in the science enclosure. If close enough, the titanium probe can draw gas directly from an active fumarole or the magma lake. Gases drawn through the probe can either be collected in evacuated bottles, analyzed with a gas chromatograph, drawn through filters, or analyzed with a quartz crystal microbalance.

The gas chromatograph (GC) can perform real-time 
analysis of gas composition. Researchers receive immediate information about gas chemistry. By collecting samples at different locations, the GC can map the change in chemistry over time and position in the crater. The physical samples are redundant with the GC but can also be analyzed in greater detail in the laboratory.

The filters are used to sample fine particulates (aerosols) suspended in the volcanic plume. At various points during the exploration of the crater, a filter sample can be taken. In parallel with the filter system, the quartz crystal microbalance (QCM) measures total mass of airborne particulates. It consists of paired quartz plates, exposed to the atmosphere, that both resonate at the same frequency until mass is collected; then the frequency of the top plate changes and the frequency difference is proportional to the collected mass. The QCM can measure the change in aerosol content over time and position during the course of the exploration.

A gamma ray spectrometer (GRS) is also included in Dante's science package. A GRS measures the radioactivity of materials. By recording and analyzing the gamma ray spectrum of a material, the quantity and type of the isotopes can be identified. The GRS can measure the increasingly younger lavas that would be encountered as the magma lake is approached. On the crater floor, the GRS can be used to analyze sublimates that quickly drop out of the gases as they exit vents and hit the cold air.

On the pan-tilt platform atop its sensor mast, Dante carries an infrared thermocouple, a device that can measure the temperature of bodies from a distance by measuring the emitted infrared radiation. The infrared thermocouple can be pointed at the magma lake whenever possible in hopes of observing a large gas bubble breaking the surface of the lake and revealing the liquid magma just beneath.

\section{Dante Software}

The design of the legged mechanism, complex or not, can clearly be achieved. The slow, smooth motion of legged devices is advantageous in rough terrain where caution (and, sometimes, sensing) limits the speed of wheeled and legged mechanisms alike. The significant remaining drawback to legs has been the difficulty in developing software to control walking. For a slow-moving walker, this challenge is not in moving the individual legs, as this is directly analogous to well-understood manipulator control, but in the coordination of leg and body motions. The legged robot, either with a realtime controller, low-level planner, or as an artifact of its architecture, must generate a sequence of leg and body motions, a gait, that will propel it along some path. We chose to control the gait with deliberative planners and to enable Dante to sense its environment so that the planners can autonomously control the robot's action.

\section{Architecture}

The architecture of Dante's software system reflects a sense-plan-act cycle that enables Dante to walk and to operate autonomously. (See Figure 7.) Ter-

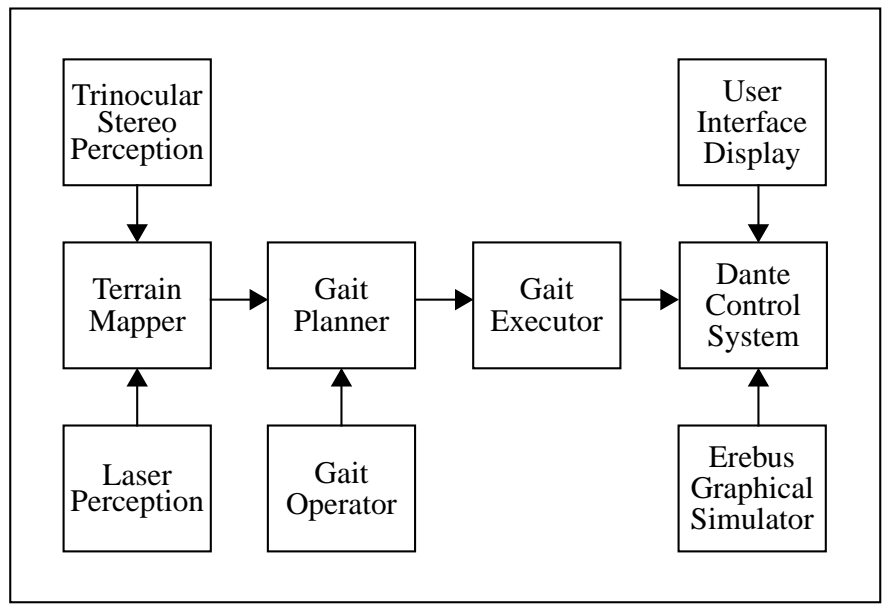

Figure 7: Dante's software architecture showing information flow

rain is sensed by both trinocular stereo and laser rangefinder perception. However, Dante can be teleoperated without its perception sensors and walk blindly by relying on human operators to direct its actions. Each perception module transforms raw sensor data into a depth map. The terrain mapper then transforms depth maps acquired in the coordinate system of a particular sensor into a common elevation map. Extensive map merging is not performed because the gait planner periodically requests maps in the current local coordinate system and there will likely be a single most recent map.

The gait operator gives instructions to walk, roll, pitch, yaw and lift (and lower) the robot. These instructions are in the form of desired trajectories to follow. The gait planner takes these trajectories and breaks them down: first it plans all the body motions required to follow the trajectory. Then, if Dante is operating autonomously, the body motion is adjusted to avoid collision with the terrain while still remaining as low as practical for stability. Next, the basic leg motions required to propel the body along the trajectory are generated. If Dante is not walking blindly, the leg motions are adjusted to clear obstacles during the recovery phase and to ensure contact with the terrain during support 
phase. This planning process, termed gait generation, results in a plan to make Dante walk along the commanded trajectory.

The gait plan is then passed to the gait executor. The executor monitors current robot state and feeds the commands to execute the gait plan to the Dante control system. The gait executor monitors progress looking for errors in the execution of the plan. When a substantial error occurs, the executor stops execution of the gait plan, determines the current robot state, and sends a message to the gait planner to initiate generation of a new plan beginning from the current location.

The Dante control system, or controller, is a realtime process that runs on-board the robot. It coordinates the actuators and reads status from encoders and other sensors. The Erebus graphical simulator acts as a virtual robot simulating all sensors including the laser rangefinder and all actuators. Its message interface is identical to that of the actual controller so plans generated by the gait planner can be previewed on the simulator to observe their outcome. The simulator is invaluable to the development of other modules because they can be tested without needing, and before the completion, of the actual sensors or actuators.

The user interface display periodically polls the controller for status information about the robot. It displays a 3D kinematic view of the robot with readouts of all the actuators and sensors. The user controls Dante's actions by giving commands that go to the gait operator.

In future implementations of the system, the gait executor could be more capable of correcting minor problems with the execution of gait plans rather than just detecting errors. Future development of this system would eliminate the gait operator process replacing it with a trajectory planner which could automatically perform the task of identifying the best route for the robot and breaking it into trajectories for the gait planner.

\section{Perception}

Trinocular stereo perception uses a new sum of sum of squared differences (SSSD) algorithm that can quickly generate depth maps of the visible terrain.[3][4] Any stereo vision system operates on the principle that the known baseline between cameras and the relative position of a feature in each camera image can be used to calculate the distance to the feature. Three cameras (trinocular) are used because depth triangulation is more accurate with longer baselines but feature correspondence is less difficult with shorter baselines. The trinocular jig combines both long and short baselines.

Maps using the rangefinder produce a donut-like image of the terrain. The rangefinder's laser beam is bounced off a spinning mirror that slowly nods. The distance to each point is measured and transformed into Cartesian coordinates creating an elevation map. In Figure 8 the robot is in the open center and an obscured sector extends upward. In this map, from the scan in Figure 6, the outer slopes of the crater are to the left and the crater drops sharply to the right.

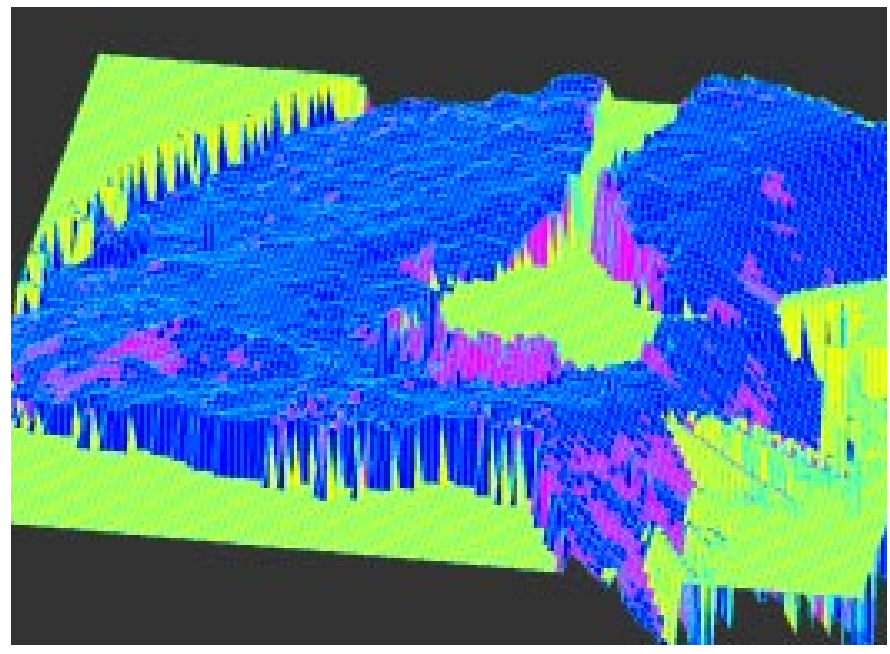

Figure 8: Terrain map generated from rangefinder data of the crater rim

Each system has strengths and weaknesses. The stereo system has a large range and very high resolution but is susceptible to lack of texture in the scene, and resolution comes at the expense of processing time (about 10 seconds for a $5 \mathrm{~cm}$ resolution map of the near-terrain). A number of tests were perform on sparsely featured terrain and on all but the smoothest blanket of snow, it performed well. The laser system has high accuracy and speed (scan acquisition is 3-5 seconds and transformation another second) but is delicate and sensitive to variations in terrain type. The locus algorithm can be applied to this terrain to smooth discrepancies and identify range shadows.[5] We felt that the two systems would combine to provide good perception results in all expected terrain and weather conditions.

\section{Planning}

Like our work on the Ambler, we have taken a primarily deliberative approach to gait planning for Dante.[6] However, plans are executed separately from their generation, allowing monitoring of their 
progress. At this point, the monitor performs only error detection not reactive error correction.

Dante's gait generation software utilizes various constraints, such as kinematics, terrain, and stability, to constrain the range of possible moves. It then orders the remaining moves so that search or optimization can be used to select the best gait to achieve the goal.

There are two basic problems with searching in constraint space for an optimal gait. First, the space grows exponentially with the number of degrees-offreedom in the robot and is more difficult to search with increasing numbers of constraints.[7] The search space can be kept manageable by considering only a small number of placements for each leg and body move. Second, the horizon effect for limited search implies that successfully finding a gait to an intermediate goal is no guarantee of reaching the ultimate goal. Unlike the Ambler, which can move any leg at any time, Dante with two fixed frames of legs has a very limited set of possible moves. This greatly simplifies the planning problem reducing it primarily to one of optimizing stability and adaptation to the terrain.[8]

Terrain is used to constrain the motions of the mechanism. As the body moves forward, lifting and lowering to maintain appropriate terrain stand-off, the leg extensions must adjust to avoid obstacles and then to make contact with the ground at footfall. The gait planner interacts with the terrain mapper to learn about the terrain surrounding the mechanism.

For stability, constraints of static stability, such the conservative support polygon, are not relevant since the mechanism is always statically stable.[9] With respect to dynamic stability measures, such as the energy stability constraint, Dante is very stable on flat terrain.[10] In Figure 9 is a graph of Dante's nominal energy stability, the energy required to tipover as a function of the position of center-of-gravity. On sloped terrain, proper control of tether tension assures that Dante's stability appears the same to the gait planner. In fact, with proper tether control increasing slope appears to the software system as simply decreasing weight. The magnitude of the energy required to tip over decreases linearly until Dante is on a vertical slope and the tipover energy is everywhere zero. However, Dante remains stable, hanging on its tether.

\section{Control}

The controller is divided between two real-time processor boards on-board the robot. One processor

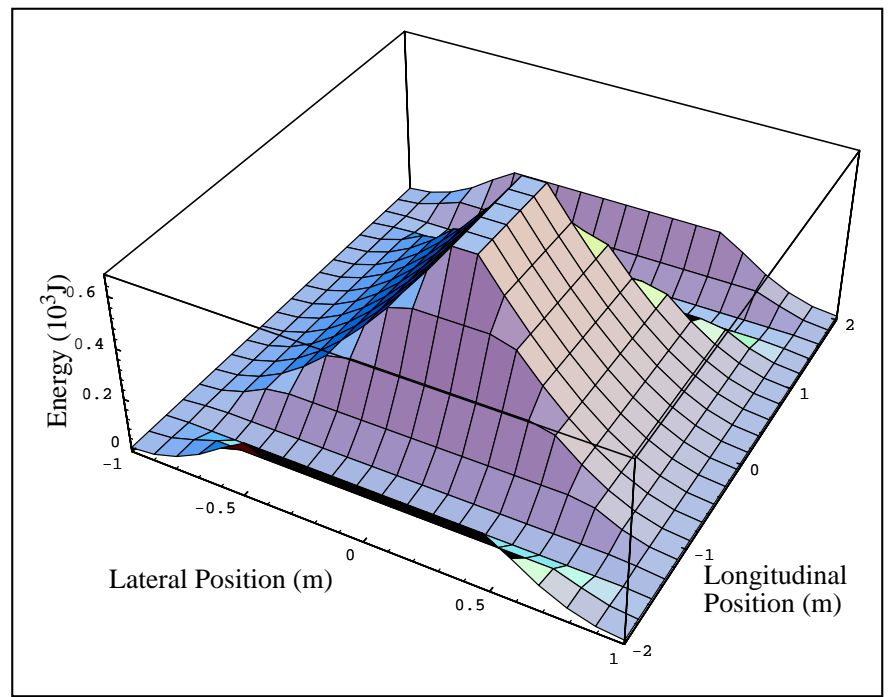

Figure 9: Dante's energy stability as a function of center-of-force position; feet are at $1.75 \mathrm{~m}$ from the longitudinal axis and $1.5 \mathrm{~m}$ from the lateral axis

is for communication and the other is for servo control. The communication processes receive motion commands and send sensor information to other software modules. The servo processes control the motions of the vertical actuators in feedback loops and send signals to motion control board that control the four-bar linkages.

The controller is designed to perform continuous walking, so requests are received, processed and queued concurrent with motion execution. The processing involves coordinating the transition between support and recovery phases and setting speeds so that legs arrive at the right place at the right time. As long as something is in the queue Dante keeps walking. The controller also checks the health of all physical devices including itself. It sends warning messages to the operator when problems are detected and recovers from those errors that it can. The controller communicates with other software modules using the TCX communications system.[11]

\section{Communication}

Using TCX, a task communications package based on experience with the Ambler's Task Control Architecture (TCA), Dante has an integrated a software system that researchers can observe, instruct, and modify during operation.[12] Processes including perception, planning, and control communicate point-to-point via messages. TCX abstracts the coding, transmission, and decoding of messages making inter-process communication look like intraprocess procedure calls. TCX has greatly reduced the time to integrate a software system and 
allowed researchers to quickly add new modules when necessary.

\section{Testing}

During the last third of the project, after spending the first third designing and the second assembling, we began a testing program for Dante. First we performed a number of component tests including the legs, feet, tether reel, electronics and fiber optic telemetry. In one tether test, we discovered when the tether was placed under tensile loads the internal fiber was so constricted that the signal was attenuated beyond reconstruction. This was unexpected as the fiber was specified to function while supporting Dante's full weight. Time precluded manufacture of a new tether so a second passively deployed communication fiber was added to the system.

Once fully assembled we undertook full system tests. Indoor flat-terrain tests were performed to verify control software. Outdoors, at a local slag heap, we performed tests walking on rough ground. During one test, while attempting to transition onto a slope, an extreme side-loading condition, four legs simultaneously broke. Ultrasonic inspection revealed that the welding in the legs, performed by an outside contractor, was substandard. The legs were subsequently repaired and reinforced.

We demonstrated fully-autonomous walking using perception in natural terrain. Finally, we performed a number of teleoperated tests while walking under tether tension control, ascending and descending artificial ramps and natural hillsides. The slope materials included hard rock, soft sand, conglomerated gravel and loose blocks, which, in retrospect, are very good approximations of the terrain Dante encountered just beneath Erebus' rim. Dante was able to succeed in all these test environments. Having completed all the testing that our 10 month time-frame would allow and with a number of problems revealed and corrected, the decision was made that we proceed to Erebus.

\section{Expedition to Erebus}

In Pittsburgh, Dante was disassembled and packed along with support equipment into crates and flown to New Zealand where we were waiting to travel on to Antarctica. On December 15, 1992 we arrived via LC-130 airplane in McMurdo Station with all equipment intact.

The first week of the expedition was occupied entirely by logistics. Upon arriving in McMurdo the group attended numerous orientations including topics of facilities, food, equipment, cargo, helicopters, safety. Everyone attended mandatory field safety training to learn everything from glacier travel to snow shelters and cooking stoves to Skidoo driving. During this period we repacked all our cargo into loads suitable for helicopter transport to Mount Erebus. This year McMurdo linked to the Internet via satellite so we were able to communicate directly, though intermittently, to Carnegie Mellon. With the cargo and people ready to go we encountered our first weather delay-helicopters were unable reach the acclimatization camp on the Fang Glacier because of cloud cover.

Two days later, on December 21, everyone in the party was flown into the Fang Glacier camp for acclimatization to altitude. No one in our party felt more than mild headaches and nausea and after two nights, we helicoptered to the Erebus Camp. The cargo began to arrive immediately. The first task was to assemble Geryon, Dante's transport cart. Dante, which is dimensioned to just fit inside the UH-1N helicopter, was then unloaded onto Geryon. We set up the mountainside control station inside an existing hut. Computer, video monitors, TDRSS communications electronics, an INMARSAT station, and Dante were moved inside and assembled into robot and control station. We tested individual components again and then took Dante for a walk outside the control station to see that the mechanism and electronics still worked. We collected data with the stereo and rangefinder and with correct filtering both systems produced high quality maps. We performed a cold-boot test overnight, shutting Dante down and allowing it to reach the ambient temperature before starting its automatic warm-up and boot cycle. After Dante had booted, we experienced our first Antarctic storm and hurried to put Dante back inside to do the final sealing and science payload preparations. We also discovered a new problem with the tether level-wind mechanism which lays the tether uniformly on the reel. It appeared that the cold temperature caused it to bind up. An additional heating unit was installed.

On December 28, we began transporting Dante to the rim of the outer crater. We started by pulling Geryon, loaded with Dante and generators, by a team of Skidoos across the first kilometer of snow fields and shallow valleys. When the grade became so steep that we could no longer Skidoo, we set anchors to which we attached Geryon's winch cable. On this day the first of many TDRSS events occurred and we broadcast video about Erebus and the progress to date. After two more days of winching and another storm interruption Dante arrived safely at the rim. The $2 \mathrm{~km}$ fiber optic line extension 
was run back to the control station and Dante was booted and operational. December 31 brought two exciting events. First a runaway Skidoo with frozen throttle went careening off towards the TDRSS dish. Although it had driven across a fiber optic line, there was no damage. On the rim, Dante walked off Geryon and into launch position. But then the volcano experienced a minor eruption. With a low roar, Erebus began tossing lava bombs from the magma lake into the outer crater. Although only "Pele's Hair", fine glass strands, reached the rim, a dense plume curtailed work for the rest of the day.

On January 1, the sensor mast was reinstalled and the science payload activated. The level wind mechanism which was still not functioning properly, was removed meaning that the tether would not be laid evenly into the reel. Although a risk, it was felt that the natural slewing of the body with the terrain would be sufficient to distribute the tether and that the large reel could function without a uniform wrap. It is interesting to note that the problem with the level wind was later identified as a loose acme screw, not temperature as was believed at the time-both could have caused it to "freeze". During the rim checkout Dante was teleoperated from Goddard. The on-board controller executed commands from Goddard operators to point, focus and zoom Dante's teleoperation cameras. The launch platform with Dante in place was then tipped over the edge of the crater. A new problem, this one with the balance of the teleoperation camera was discovered. Apparently the panning actuator could not swing the heavy eccentric load of the sealed camera. This was corrected by adding a counterbalancing weight, a carabiner. Dante was launched and the descent began with Dante rappelling $6.5 \mathrm{~m}$ to about $10 \mathrm{~m}$ below the rim. (See Figure 10.)

On January 2, digital communication to Dante was lost and the video signal greatly degraded. We discovered that the passively-deployed fiber optic line, which had stiffened in the cold, was tangling as it unspooled. We went to the rim and cleared the tangles, restoring the digital communication, but found that as the robot proceeded more tangles would occur. The decision was made to try to unspool the fiber at the current position and feed it down from the rim rather than risk more problems once Dante was out of reach. During this operation a number of twists and bends were corrected. Eventually, a sharp bend unspooled, twisted and severed the fiber. Hope of repairing a single-mode fiber under existing conditions was non-existent so the mission was stopped

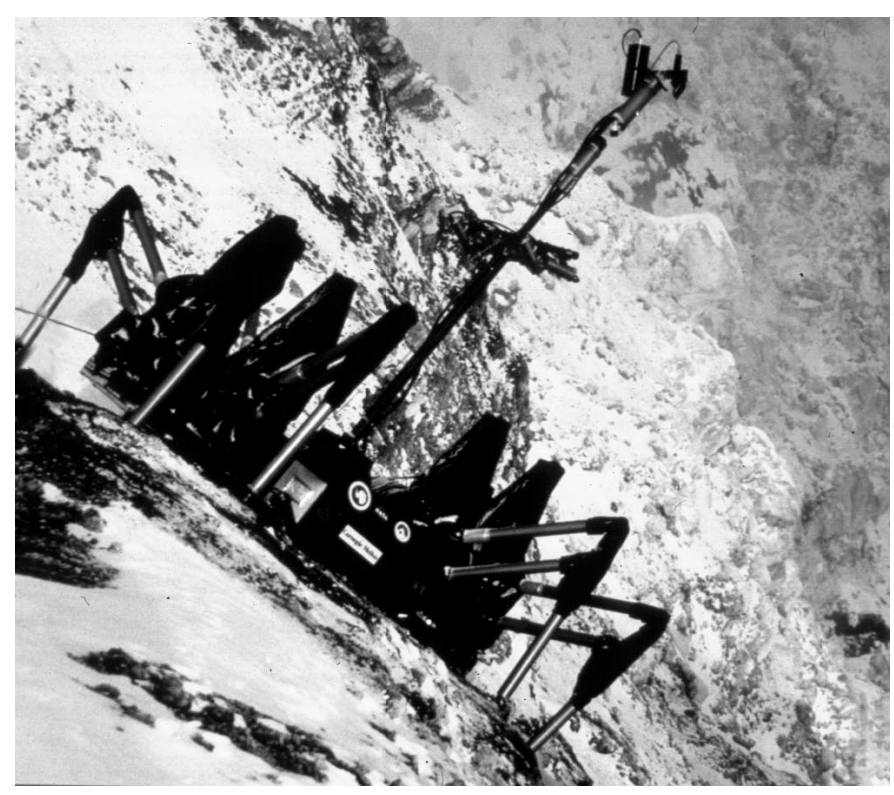

Figure 10: Dante on Mount Erebus' outer crater wall

at that point. We held a live news conference over the TDRSS link and explained what had happened. We had great success but were stopped short of the crater floor.

The next few days were spent dragging Dante back to the rim with Geryon's winch, loading the cart and winching everything back to the hut for disassembly and repacking. Equipment and people were shipped back to McMurdo Station where we prepared equipment for sea transport and waited for planes back home.

\section{Summary}

What went right:

- Created and partially demonstrated a credible robot and exploration mission

- Successfully tested remote-controlled robot in harsh climate

- Walked with intrinsic gait

- The robot scale and capabilities were correct for the terrain

- The software architecture was flexible and robust

- Sealed computers, electronics and telemetry worked

- Satellite system and TCX enabled live remote video and teleoperation

What went wrong:

- Didn't reach the bottom of the crater.

- Moreover, didn't descend far enough to collect any gas samples.

- Dante didn't walk as fast or as rigidly as 
intended

- Sensors including the tension sensor and inclinometers required frequent recalibration

- As many contingencies as were covered, we didn't test enough get everything right so some components, like the level wind, caused problems and others, like the fiber, just broke.

The Dante project demonstrated further development in mobile robotics, showed the potential of exploratory robots in the future and, because of the close analogy to the Antarctic environment, discovered important challenges in future planetary exploration.

Dante provides fundamental insights into the design, construction and control of environmentally survivable, capable systems. Novel designs simplify mechanical systems and provide viable solutions to exploration in extremely rugged terrain. The perception component of our research advances the state-of-art in mapping rugged terrain covered with difficult materials like ice and snow. The approaches used for modeling the sparsely-featured terrain and objects encountered in the Mount Erebus mission are applicable to a wide range of navigation and manipulation tasks. The problems of systems integration, systems architecture, and coordination of multiple, interacting software components are faced by every robotics program. In particular, issues of extended autonomy, long-distance and long-delay telemetry, and high-reliability software must necessarily be addressed to deploy robots in Antarctica.

This program is applying advanced robotics technologies, research robots and expertise developed and validated in the laboratory that are essential for future missions in space and on Earth.

\section{Acknowledgment}

The development of Dante was supported by the National Aeronautics and Space Administration under grant NAGW-1175. The expedition to Mount Erebus was supported in part by the National Science Foundation under grant DPP-9118056 for Antarctic science project S-081. We would like to thank the pilots and crew of the U.S. Navy VXE-6 for helicopter support.

This paper will appear in a special issue of Robotics and Autonomous Systems under the title "Exploring Mount Erebus by Walking Robot."

\section{References}

[1] G. Zreda-Gostynska, P. Kyle, D. Finnegan, Chlorine, Fluorine, and Sulfur Emissions from
Mount Erebus, Antarctica and Estimated Contributions to the Antarctic Atmosphere, submitted to Geophysical Research Letters (1993).

[2] J. Bares, Configuration of Autonomous Walkers for Extreme Terrain, Ph.D. Dissertation in Civil Engineering, Carnegie Mellon University, May 1991.

[3] M. Okutomi, T. Kanade, A Multiple-Baseline Stereo, Proceedings of IEEE Conference on Computer Vision and Pattern Recognition, 1991.

[4] B. Ross, A Practical Stereo Vision System, Proceedings of IEEE Conference on Computer Vision and Pattern Recognition, 1993.

[5] I. Kweon, T. Kanade, High Resolution Terrain Maps from Multiple Sensor Data, IEEE International Workshop on Intelligent Robots an Systems, July 1990.

[6] D. Wettergreen, C. Thorpe, Gait Generation for Legged Robots, IEEE International Conference on Intelligent Robots and Systems, July 1992.

[7] J-C. Latombe, Robot Motion Planning, Kluwer Academic, 1991

[8] J. Bares, W. Whittaker, Walking Robot with a Circulating Gait, IEEE International Workshop on Intelligent Robots an Systems, July 1990.

[9] S. Mahalingam, W. Whittaker, Terrain Adaptive Gaits For Walkers with Completely Overlapping Work Spaces, Robots 13, May, 1989, pp.1-14.

[10] D. Mesurri, C. Klein, Automatic body Regulation for Maintaining Stability of a Legged Vehicle During Rough-Terrain Locomotion, IEEE Journal of Robotics and Automation, Vol. RA1, No. 3, September, 1985, pp.132-141.

[11] D. Christian, Dante Controller, Robotics Institute Technical Report, Carnegie Mellon University, 1993.

[12] C. Fedor, TCX Reference Manual, Robotics Institute Technical Report, Carnegie Mellon University, 1993. 